\title{
Generalized Geometry-Based Optimal Power Control in Wireless Networks
}

\author{
Wei Wang ${ }^{\dagger}$, Kang G. Shin ${ }^{\ddagger}$, Zhaoyang Zhang ${ }^{\dagger}$, Wenbo Wang ${ }^{\S}$, Tao Peng ${ }^{\S}$ \\ ${ }^{\dagger}$ Department of Information Science and Electronic Engineering \\ Zhejiang Provincial Key Laboratory of Information Network Technology \\ Zhejiang University, Hangzhou 310027, P.R. China \\ ${ }^{\ddagger}$ Department of Electrical Engineering and Computer Science \\ The University of Michigan, Ann Arbor, MI 48109-2121, U.S.A. \\ $\S$ Wireless Signal Processing and Networking Lab \\ Beijing University of Posts and Telecommunications, Beijing 100876, P.R. China \\ Email:wangw@zju.edu.cn
}

\begin{abstract}
Geometry-based optimal power control was proposed in [14] to transform the power-control problem to a new geometrical problem on the position relationship between a line and some points. This scheme provides a novel visual perspective and lowers the complexity of optimization. We generalize this scheme to a larger class of power-control optimization problems so as to maximize the network utility with multiple average and peak power constraints in wireless networks. To facilitate the handling of the geometrical model, we define a subset of geometrical models with specified characteristics, called a regular geometrical model, and derive the type of power-control problems eligible for the regular geometrical model. For such a type of problems, two strategies are proposed for the construction of the regular geometrical model. Utilizing geometrical properties, we propose a novel geometry-based optimization scheme for the general power-control problem. Its computational complexity is significantly lower than the conventional algorithms. We also provide a further discussion on irregular geometrical model cases. Finally, we provide two examples of deploying the proposed geometry-based power-control scheme.
\end{abstract}

\section{INTRODUCTION}

Power control plays a key role in interference coordination and energy management in wireless networks. In case of voice communications, for example, power control is used to meet the users' required signal to interference plus noise ratios (SINR) [1]. Each user adjusts its transmit power just to meet the SINR requirement. A unified framework is proposed in [2] for the convergence conditions of distributed power-control algorithms.

For data communications, a user can increase its transmit power to achieve a higher data rate by using adaptive modulation and coding schemes [3]. The power-control framework proposed in [2] is extended for data communications in [4]. In these papers, power control was used to combat the interference among the users and achieve high throughput in wireless networks.

This work is supported by National 973 Program under grant No. 2009CB320405, National Natural Science Foundation of China under grant No. 61001098, 60972057, 60972058, and National Science and Technology Specific Major Projects under grant No. 2012ZX03002009.
Over the recent decades, there have been various techniques proposed to mitigate the interference. In the presence of internode interferences, the Successive Interference Cancelation (SIC)[5] at the receivers is an efficient approach for multiple simultaneous transmitters. For interference-free transmissions, Orthogonal Frequency Division Multiplexing (OFDM) [6] provides orthogonal subcarriers without inter-carrier interference. Multiple-Input and Multiple-Output (MIMO)[7] can also provide multiple parallel channels by decomposing the channel matrix between the antennas of the transmitter and the receiver. In these scenarios mentioned above, the achievable total throughput would be an increasing and concave function of its transmit power according to the Shannon's capacity formula.

As the objective functions of most power-control problems in wireless networks are concave, numerous researchers [8], [9], [10] adopted convex optimization, which is an effective mathematical tool to minimize a convex function subject to convex constraints. There are two types of power-control optimization. The first is to approach the optimum based on Lagrangian duality by numerical search methods, such as the steepest descent method [11], [12]. The complexity of this type of optimization depends on the initial point and the required accuracy of optimal solution. Also, the complexity increases significantly with the number of Lagrangian multipliers. The second type of optimization is to obtain the best directly by comparing the objective values for all possible optimal solutions, i.e., the vertices of the convex feasible region [13]. By this type of algorithms, the optimal solution can be obtained deterministically, and its complexity depends mainly on the number of possible optimal solutions.

In our earlier work [14], a geometrical model was constructed to transform a power-control problem to a new geometrical problem on the position relationship between a line and multiple points. This geometrical model is used to obtain an optimal solution by comparing possible optimal solutions, which belong to the second type of optimization mentioned above. The geometrical properties are utilized to reduce the number of possible solutions, thus lowering the computational 
complexity. Note that the algorithm in [14] was designed only for the special optimization problem. This motivates us to study how to deploy the geometry-based scheme for more general power-control problems, and the type of problems for which the geometry-based scheme is suitable.

In this paper, we generalize the geometry-based optimal power-control scheme in [14] and establish a new framework that can be applied to a much larger class of algorithms in wireless networks, including multiple access channels, broadcast channels, and multiple parallel channels with the consideration of multiple average and peak power constraints. The main contributions of this paper are as follows.

- A class of power-control optimization problems can be transformed to geometrical problems on the position relationship between a line and some points, which provide a novel visual and intuitive perspective of power-control optimization.

- The geometrical model is easier to handle if it is regular. ${ }^{1}$ We propose two strategies for constructing regular geometrical models and derive two conditions to determine if the power-control problem is eligible to be modeled as a regular geometrical model.

- We develop the optimization methods for both regular and irregular geometrical models. Especially, for the regular case, it reduces the computational complexity significantly as compared to the conventional algorithms. In addition, both multiple access channels and multiple parallel channels are discussed as deployment examples of the proposed geometry-based power-control scheme.

The rest of this paper is organized as follows. Section II describes the problem formulation. In Section III, the powercontrol optimization problem is modeled as a geometrical model. Section IV investigates the regularity of geometrical models. Section V proposes a geometry-based optimal powercontrol scheme. Irregular geometrical models are discussed in Section VI. Two deployment examples are provided in Section VII. Finally, the paper concludes with Section VIII.

\section{Problem Formulation}

Consider a general power-control optimization problem in which $N$ users communicate with their respective receivers. Let $\mathcal{N}$ be the set of users. Independent variables of the optimization problem are the power vector $\mathbf{P}=$ $\left\{P_{1}, P_{2}, \cdots, P_{N}\right\}$, where $P_{i}$ is user $i$ 's transmit power.

The objective of general power-control optimization is to maximize the long-term network utility $\mathbb{E}[U(\mathbf{P})]$ by adjusting $\mathbf{P}$, where $\mathbb{E}(\cdot)$ is the mathematical expectation. The utility $U(\mathbf{P})$ increases and the marginal utility $\partial U(\mathbf{P}) / \partial \mathbf{P}$ decreases with the increase of transmit power in a lot of scenarios. It is assumed in this problem that $U(\mathbf{P})$ is an increasing and concave function of $\mathbf{P}$. The network utility $U(\mathbf{P})$ can be one of many functions, such as the weighted sum-rate for multiple access channels, broadcast channels and multiple parallel channels.

\footnotetext{
${ }^{1}$ The definition of regular geometrical model will be provided in Section IV.
}

In the optimization problem, there are two types of linear constraints: $J$ average and $K$ peak power constraints. The constraints can be set to the transmit power limit for an individual user, the total transmit power limit for all users, and the interference at some measurement points, in average and/or peak sense. The power-control optimization problem can be formulated as

$$
\begin{array}{ll}
\max & \mathbb{E}[U(\mathbf{P})] \\
\text { s.t. } & P_{i} \geq 0, \quad \forall i \in \mathcal{N} \\
& \mathbb{E}\left[\sum_{i} a_{i j} P_{i}\right] \leq c_{j}, \quad \forall j \in\{1,2, \cdots, J\} \\
& \sum_{i} b_{i k} P_{i} \leq d_{k}, \quad \forall k \in\{1,2, \cdots, K\}
\end{array}
$$

where $a_{i j}$ and $b_{i k}$ are the random coefficients of linear constraints, $c_{j}$ and $d_{k}$ are the limits of constraints. It is assumed that the probability density functions (p.d.f.) of $a_{i j}$ and $b_{i k}$ are known. All of the coefficients $a_{i j}, b_{i k}, c_{j}$ and $d_{k}$ are assumed to be greater than, or equal to 0 .

\section{Geometrical ANALYsis}

\section{A. Lagrangian Optimality}

The above general power-control problem takes into account both average and peak power constraints. We first treat the average power constraints by the Lagrangian optimality and divide the original problem into multiple subproblems for each snapshot, which is an instant with a sample of random coefficients $a_{i j}$ and $b_{i k}$.

Let $\lambda=\left\{\lambda_{1}, \cdots, \lambda_{J}\right\}$ be the vector of the Lagrangian multipliers corresponding to the average power constraints. The optimal solution of the power-control problem can then be obtained if and only if there exist $\lambda$ and a power-control scheme $\mathbf{P}$ such that the power $\mathbf{P}$ is a solution of the optimization problem for each snapshot [15]:

$$
\begin{array}{ll}
\max & U(\mathbf{P})-\sum_{j} \lambda_{j}\left(\sum_{i} a_{i j} P_{i}-c_{j}\right) \\
\text { s.t. } & \lambda_{j}\left(\mathbb{E}\left[\sum_{i} a_{i j} P_{i}\right]-c_{j}\right)=0, \forall j \in\{1,2, \cdots, J\} \\
& \sum_{i} b_{i k} P_{i} \leq d_{k}, \forall k \in\{1,2, \cdots, K\} .
\end{array}
$$

Let $L$ denote the Lagrangian function for each snapshot and $\beta=\left\{\beta_{1}, \beta_{2}, \cdots, \beta_{K}\right\}$ be the vector of the Lagrangian multipliers corresponding to the peak power constraints. According to the Karush-Kuhn-Tucker (KKT) condition [11], the above problem is transformed to:

$$
\begin{aligned}
\max L=U(\mathbf{P}) & -\sum_{j} \lambda_{j}\left(\sum_{i} a_{i j} P_{i}-c_{j}\right) \\
& -\sum_{k} \beta_{k}\left(\sum_{i} b_{i k} P_{i}-d_{k}\right)
\end{aligned}
$$




$$
\begin{array}{ll}
\text { s.t. } & \lambda_{j}\left(\mathbb{E}\left[\sum_{i} a_{i j} P_{i}\right]-c_{j}\right)=0, \forall j \in\{1,2, \cdots, J\} \\
& \beta_{k}\left(\sum_{i} b_{i k} P_{i}-d_{k}\right)=0, \forall k \in\{1,2, \cdots, K\} .
\end{array}
$$

Note that the Lagrangian multipliers $\lambda$ and $\beta$ have different features. $\lambda$ is adjusted to meet the average power constraints, and hence $\lambda$ is unique for all snapshots. Unlike $\lambda$, the Lagrangian multiplier $\mathbb{\beta}$ for peak power constraints is determined for each snapshot to ensure the peak power constraints are satisfied for all snapshots, which indicates the difference between average constraints and peak constraints.

Because of the inherent feature of $\lambda$ and $\beta$, the Lagrangian multipliers should be adjusted hierarchically. $\lambda$ is adjusted for average power constraints and is unique for all snapshots. In the subproblem of each snapshot, the independent variables $\mathbf{P}$ and $\beta$ are adjusted for optimization with given $\lambda$.

To obtain the optimality condition, we calculate the partial derivative of the Lagrangian function $L$ with respect to $P_{i}$ as

$$
\frac{\partial L}{\partial P_{i}}=\frac{\partial U(\mathbf{P})}{\partial P_{i}}-\sum_{j} \lambda_{j} a_{i j}-\sum_{k} \beta_{k} b_{i k} .
$$

Let $P_{i}^{*}$ be user $i$ 's optimal power and $P_{i}^{\max }$ be the user $i$ 's maximum power restricted by the peak power constraints, which can be calculated as $P_{i}^{\max }=\min \left(d_{k} / b_{i k}\right)$. For the users transmitting data with $P_{i}^{*}>0$, the optimality condition is specified by the following theorem.

Theorem 1: In the general power-control optimization problem, the sufficient and necessary optimality condition is that $\partial L / \partial P_{i}=0$ for the users transmitting with $P_{i}^{*}>0$ and $\partial L / \partial P_{i}<0$ for the users who are not transmitting.

Proof: Since the network utility $U(\mathbf{P})$ is an increasing and concave function of $P_{i}$, the following condition is always met:

$$
\frac{\partial^{2} L}{\partial P_{i}^{2}} \leq 0
$$

According to this equation, if $\partial L / \partial P_{i} \leq 0$ when $P_{i}=0$, then $\partial L / \partial P_{i} \leq 0$ for any $P_{i} \in\left[0, P_{i}^{\max }\right]$, so the objective is maximized when $P_{i}=0$. Similarly, if $\partial L / \partial P_{i} \geq 0$ when $P_{i}=P_{i}^{\max }$, then $\partial L / \partial P_{i} \geq 0$ for any $P_{i} \in\left[0, P_{i}^{\max }\right]$, so the objective is maximized when $P_{i}=P_{i}^{\max }$. If $\partial L / \partial P_{i}>0$ when $P_{i}=0$ and $\partial L / \partial P_{i}<0$ when $P_{i}=P_{i}^{\max }$, there must exist a point $P_{i} \in\left(0, P_{i}^{\max }\right)$ such that $\partial L / \partial P_{i}=0$, which is the optimal solution in this case.

Based on the above analysis, the optimal power $P_{i}^{*}$ is achieved at either local maxima or the vertices of its feasible region. Because of the increasing and concave objective function, the sufficient and necessary optimality conditions of the general power-control problem are:

$$
\begin{cases}\left.\frac{\partial L}{\partial P_{i}}\right|_{P_{i}=0} \leq 0 & \Rightarrow P_{i}^{*}=0 \\ \left.\frac{\partial L}{\partial P_{i}}\right|_{P_{i}=P_{i}^{*}=0} & \Rightarrow 0<P_{i}^{*}<P_{i}^{\max } \\ \left.\frac{\partial L}{\partial P_{i}}\right|_{P_{i}=P_{i}^{\max } \geq 0} & \Rightarrow P_{i}^{*}=P_{i}^{\max }\end{cases}
$$

When $P_{i}^{*}=P_{i}^{\max }$, the Lagrangian multiplier $\beta$ can be adjusted for each snapshot to make $\partial L / \partial P_{i}=0$ when
$P_{i}=P_{i}^{\max }$. Therefore, the optimality condition for the users transmitting with $P_{i}^{*}>0$ is $\partial L / \partial P_{i}=0$.

\section{B. Geometrical Model}

To provide a visual and intuitive perspective, we propose a novel geometrical model to transform the general powercontrol optimization problem to a new geometrical problem associated with the position relationship of points and lines.

According to Theorem 1 and Eq. (11), the optimality condition of the transmitting users in the general power-control optimization problem can be rewritten as an expression of a line in a two-dimensional space.

$$
\frac{\partial L}{\partial P_{i}}=A x_{i}+B-y_{i}=0
$$

where

$$
\begin{gathered}
A x_{i}=\frac{\partial U(\mathbf{P})}{\partial P_{i}} \\
y_{i}=\sum_{j} \lambda_{j} a_{i j} \\
B=-\sum_{k} \beta_{k} b_{i k} .
\end{gathered}
$$

Considering the power-control problem from a geometrical viewpoint, the line is expressed as $y=A x+B$, where $A$ and $B$ are the slope and intercept of the line, respectively. Each user $i$ has a corresponding point $S_{i}\left(x_{i}, y_{i}\right)$ in a twodimensional space, where $x_{i}$ and $y_{i}$ as the horizontal and vertical coordinates of the point $S_{i}$. Define $\mathcal{S}$ as the set of all points $S_{i}, \forall i \in \mathcal{N}$.

This way, the problem is transformed to a new geometrical problem on the position relationship of points $S_{i} \in \mathcal{S}$ and the line $y=A x+B$. The geometrical model is to adjust the position relationship of the line $y=A x+B$ and the points $S_{i} \in \mathcal{S}$ to satisfy the following optimality condition.

Theorem 2: Define $\mathcal{N}_{T}$ as a set of the users whose corresponding points are on the line $y=A x+B, \mathcal{N}_{T} \subseteq \mathcal{N}$ and $\mathcal{N}_{T} \neq \phi$. The optimal solution of the geometrical model is achieved when the points $S_{i}, \forall i \in \mathcal{N}_{T}$ are on the line $y=A x+B$ and all the other points $S_{i}, \forall i \in \mathcal{N} \backslash \mathcal{N}_{T}$ are above the line.

Proof: In a two-dimensional space, since the line $y=$ $A x+B$ goes through all the points $S_{i}\left(x_{i}, y_{i}\right), \forall i \in \mathcal{N}_{T}$, and all the other points are above the line, the following should be satisfied:

$$
\begin{gathered}
y_{i}=A x_{i}+B, \forall i \in \mathcal{N}_{T} \\
y_{i}>A x_{i}+B, \forall i \in \mathcal{N} \backslash \mathcal{N}_{T} .
\end{gathered}
$$

The above equations and inequalities can be rewritten using the geometrical model Eq. (14), as

$$
\begin{gathered}
\left.\frac{\partial L}{\partial P_{i}}\right|_{P_{i}=P_{i}^{*}}=0, \forall i \in \mathcal{N}_{T} \\
\left.\frac{\partial L}{\partial P_{i}}\right|_{P_{i}=P_{i}^{*}}<0, \forall i \in \mathcal{N} \backslash \mathcal{N}_{T} .
\end{gathered}
$$


One can observe that the optimality condition in the geometrical model satisfies the optimality conditions in the general power-control problem in Theorem 1 and $\mathcal{N}_{T}$ is just the set of the users who transmit simultaneously at the snapshot.

\section{Regularity of Geometrical Models}

In a geometrical model, the positions of both the line $Y=$ $A x+B$ and the points $S_{i} \in \mathcal{S}$ are dependent on the power $\mathbf{P}$. In such a case, it is difficult ${ }^{2}$ to adjust $\mathbf{P}$ to satisfy the optimality condition of the position relationship in Theorem 2.

Here, we focus on a subset of geometrical models, called regular geometrical models.

Definition 1 (Regular Geometrical Model): A geometrical model is said to be regular if it satisfies the following two conditions: (1) the point coordinates $x_{i}$ and $y_{i}$ are fixed, and (2) the line parameters $A$ and $B$ are unique for all $i \in \mathcal{N}$. Otherwise, it is an irregular geometrical model.

Remark 1: In the regular geometrical model, the coordinates $x_{i}$ and $y_{i}$ are determinate with given $\lambda$, the positions of the points $S_{i} \in \mathcal{S}$ are fixed. In such a case, the original geometrical model can be simplified to adjust the position of only the line $y=A x+B$ by changing the independent variables $\mathbf{P}$ and $\beta$.

Obviously, not all geometrical models are regular, and hence it is necessary to investigate which type of powercontrol optimization problems can be modeled as a regular geometrical model.

Lemma 1: If $x_{i}$ is independent of $\mathbf{P}$ for all $i \in \mathcal{N}$, the positions of the points $S_{i} \in \mathcal{S}$ are fixed.

Proof: Since linear constraints are considered here, the independent variables $\mathbf{P}$ and $\beta$ do not affect the value of $y_{i}$ according to Eq. (16). Given $\lambda$ in a specified snapshot, $y_{i}$ is fixed.

$\beta$ is the vector of the Lagrangian multipliers for peak power constraints, which do not appear in the expression of $x_{i}$. Thus, $x_{i}$ will depend on the power $\mathbf{P}$ only. If $x_{i}$ is independent of $\mathbf{P}, x_{i}$ is fixed.

Based on Eq. (15), $A$ and $x_{i}$ are decomposed from $\partial U(\mathbf{P}) / \partial P_{j}$. To obtain a regular geometrical model, we must consider the following two rules when designing the decomposition strategy.

- $A$ is unique for all $i \in \mathcal{N}$ (according to the second condition of Definition 1).

- $x_{i}$ is independent of $\mathbf{P}$ (according to Lemma 1).

Using the above two rules, we design a strategy of decomposing $A$ and $x_{i}$ as follows.

Strategy 1 (Decomposition of $A$ and $x_{i}$ ):

$$
\begin{gathered}
A=\text { g.c.d. }\left(\frac{\partial U(\mathbf{P})}{\partial P_{1}}, \frac{\partial U(\mathbf{P})}{\partial P_{2}}, \cdots, \frac{\partial U(\mathbf{P})}{\partial P_{N}}\right) \\
x_{i}=\frac{\partial U(\mathbf{P})}{\partial P_{i}} / A, \forall i \in \mathcal{N}
\end{gathered}
$$

where g.c.d. $(n)$ is the greatest common divisor of $n$.

\footnotetext{
${ }^{2}$ Here, the word "difficult" is used from the computation complexity view. It is difficult if the complexity is high.
}

Remark 2: Direct calculation of the g.c.d. is not always easy when $\partial U(\mathbf{P}) / \partial P_{i}$ is not an integer polynomial. We can thus use an intuitive approach for the decomposition of $A$ and $x_{i}$. Let $\mathbf{P}_{i}^{-}$be the power vector of all but user $i$. Due to the assumed homogeneity of users, all elements of $\mathbf{P}_{i}^{-}$should have the same style in the expression of $\partial U(\mathbf{P}) / \partial P_{i}$. The difficulty of decomposition is caused by the presence of $\mathbf{P}$ in $\partial U(\mathbf{P}) / \partial P_{i}$. If the partial derivative includes only $P_{i}$ or $\mathbf{P}_{i}^{-}$, there exists $\mathbf{P}$ in $x_{i}$ that makes the geometrical model irregular. If the partial derivative includes all the elements of $\mathbf{P}$, the symmetric parts in the expression of $\partial U(\mathbf{P}) / \partial P_{i}, \forall i \in \mathcal{N}$ are decomposed into $A$ and the other parts are into $x_{i}$.

According to the intuitive method in Remark 2, the resulting $A$ is a common divisor but not always the greatest, because the constant in $\partial U(\mathbf{P}) / \partial P_{i}$ can be decomposed into either $A$ or $x_{i}$. The following lemma states that the decomposition of the constant does not affect the optimal solution.

Lemma 2: For two different decomposition results satisfying $A^{\prime}=A c$ and $x_{i}^{\prime}=x_{i} / c$ where $c$ is independent of $\mathbf{P}$ and $i$, the optimization solutions of $A-x_{i}$ decomposition and $A^{\prime}-x_{i}^{\prime}$ decomposition are the same.

Proof: From a geometrical view, if the slope of the line changes as $A^{\prime}=A c$ and the horizontal coordinates of points change as $x_{i}^{\prime}=x_{i} / c, \forall i \in N$, it is obvious that the position relationship of the line and the points does not change.

Based on the optimization conditions (18) and (19), the product of $A$ and $x_{i}$ is not affected by the decomposition strategy,

$$
A x_{i}=A^{\prime} x_{i}^{\prime}=\frac{\partial U(\mathbf{P})}{\partial P_{i}},
$$

so the optimal solutions based on these two decomposition results are the same.

Assuming adoption of the above decomposition strategy, the following lemma discusses the type of power-control problems that can satisfy the decomposition rule on $x_{i}$ for construction of the regular geometrical model.

Lemma 3: If $\frac{\partial U(\mathbf{P})}{\partial P_{i}} / \frac{\partial U(\mathbf{P})}{\partial P_{j}}$ is independent of $\mathbf{P}, \forall i, j \in \mathcal{N}$, the decomposition with Strategy 1 makes $A$ unique for all $i \in \mathcal{N}$ and $x_{i}$ independent of $\mathbf{P}$.

Proof: By the proposed decomposition strategy, $A$ should be the same for the decomposition of any $\partial U(\mathbf{P}) / \partial P_{i}, \forall i \in$ $N$, because it is the g.c.d. of these partial derivatives.

We prove the independence of $x_{i}$ from $\mathbf{P}$ by contradiction. Suppose there exists $x_{i}$ that is dependent on $\mathbf{P}$, then $x_{i}$ can be considered as the product of two factors:

$$
x_{i}=\delta_{i}(\mathbf{P}) \cdot \varepsilon_{i}
$$

where $\delta_{i}(\mathbf{P})$ is the part dependent on $\mathbf{P}$ and $\varepsilon_{i}$ is the part independent of $\mathbf{P}$.

According to Eqs. (22) and (23),

$$
\frac{\partial U(\mathbf{P})}{\partial P_{i}} / \frac{\partial U(\mathbf{P})}{\partial P_{j}}=\frac{A x_{i}}{A x_{j}}=\frac{x_{i}}{x_{j}} .
$$

Because $\frac{\partial U(\mathbf{P})}{\partial P_{i}} / \frac{\partial U(\mathbf{P})}{\partial P_{j}}$ is independent of $\mathbf{P}, x_{i} / x_{j}$ is independent of $\mathbf{P}, \forall i, j \in \mathcal{N}$. 


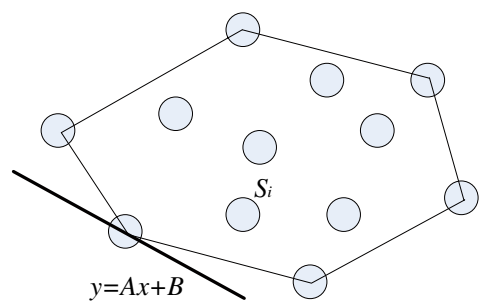

Case 1

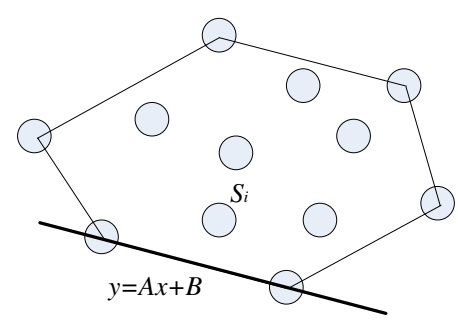

Case 2

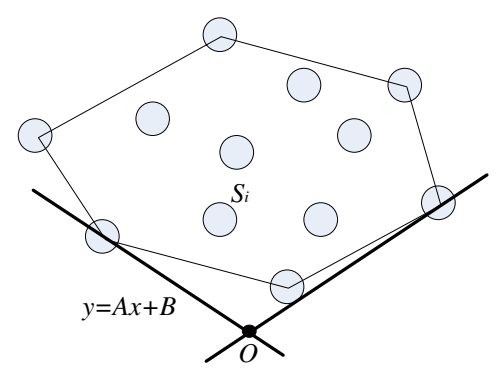

Case 3

Fig. 1. Possible optimal solutions for the regular geometrical model

As $x_{i}$ is dependent on $\mathbf{P}$ and $x_{i} / x_{j}$ is independent of $\mathbf{P}$, $v_{i}(\mathbf{P})$ should be a common factor of $x_{i}$ and $x_{j}$. Because $x_{i} / x_{j}$ is independent of $\mathbf{P}$ for all $j \in \mathcal{N}, \delta_{i}(\mathbf{P})$ is a common factor of all the partial derivatives. In that case, $\delta_{i}(\mathbf{P})$ should be a part of $A$ rather than $x_{i}$ based on Eq. (22) in the proposed decomposition strategy, which is a contradiction.

Using the proposed decomposition strategy, $A$ and $x_{i}$ are obtained for construction of the regular geometrical model. $y_{i}$ satisfies the condition of regular geometrical model because all the average power constraints are linear. The remaining problem for constructing the regular geometrical model is the condition on the intercept of the line $B$.

According to Eq. (17), $b_{i k}$ is contained in $B$, which causes different $B$ 's to be obtained from Eq. (14) for different $i \in \mathcal{N}$. Let $\mathbb{B}$ be the matrix of the coefficients $b_{i k}, \forall i \in \mathcal{N}, \forall k \in$ $\{1,2, \cdots, K\}$. We consider only one constraint first, then the corresponding coefficients are denoted as $b_{i}^{*}, \forall i \in \mathcal{N}$. In order to construct the regular geometrical model, $\mathbb{B}$ is normalized for obtaining a unique $B$ for all $i \in \mathcal{N}$.

\section{Strategy 2 (Normalization of $B$ ):}

By dividing Eq. (14) by $b_{i}^{*}$, the coefficients $b_{i}^{*}$ in the original geometrical model is considered in $x_{i}$ and $y_{i}$ instead of $B$ in the geometrical model. The parameters of the geometrical model can be written as

$$
\begin{aligned}
A x_{i} & =\frac{\partial U(\mathbf{P}) / \partial P_{i}}{b_{i}^{*}} \\
y_{i} & =\sum_{j} \frac{\lambda_{j} a_{i j}}{b_{i}^{*}} \\
B & =-\sum_{k} \beta_{k} .
\end{aligned}
$$

Lemma 4: By Strategy 2, $B$ is unique for all $i \in \mathcal{N}$ if the rank of $\mathbb{B}$ is 1 .

Proof: If the rank of $\mathbb{B}$ is 1 , all the peak power constraints are linear correlations with each other. In that case, only the strictest constraint is effective. Assume the $k$-th peak constraint is strict without loss of generality. It implies that only one line of $\mathbb{B}$ needs to be considered. There are two cases of the coefficients: (1) in the line $k$ of $\mathbb{B}$, all the $b_{i} k$ are equal for all $i$, so $B=-\beta_{k} b_{i} k$ is unique for all $i$; (2) the values of $b_{i} k$ are not equal. In such a case, some adjustments are needed to make $B$ unique. By the proposed normalization strategy, $B$ is unique for all $i \in \mathcal{N}$ for the latter case.

In summary, based on Lemmas 1, 3 and 4, the following theorem states the conditions of transforming the powercontrol optimization problems to the regular geometric model.

Theorem 3: The power-control optimization problem can be transformed to a regular geometrical model if it satisfies the following conditions:

(1) $\frac{\partial U(\mathbf{P})}{\partial P_{i}} / \frac{\partial U(\mathbf{P})}{\partial P_{j}}$ is independent of $\mathbf{P}, \forall i, j \in \mathcal{N}$,

(2) the rank of $\mathbb{B}$ is 1 .

\section{Optimal Power-Control BASEd on Regular GEOMETRICAL MODELS}

\section{A. Optimal Solution}

We propose a geometry-based optimization by utilizing the geometrical properties of the regular geometrical model. Note that the proposed algorithm can be applied for not only the general power-control optimization but also other optimizations using the regular geometrical model.

In a regular geometrical model, the positions of the points $S_{i} \in \mathcal{S}$ are fixed. For the optimal power control, only the position of the line $y=A x+B$ is adjusted. From the above analysis, $A$ is found to include the power vector $\mathbf{P}$ and $B$ to include the Lagrangian multiplier $\beta$. Since $\beta$ is configurable for each snapshot, we can adjust $\mathbf{P}$ to change the slope $A$ with an arbitrary (and appropriate) intercept $B$ such that some of the points $S_{i}, \forall i \in \mathcal{N}_{T}$ on the line and all the other points above the line.

All the points $S_{i} \in \mathcal{S}$ can construct an external polygon whose vertices are a subset of $\mathcal{S}$ and all the points of $\mathcal{S}$ are inside the polygon. To guarantee all the points either on or above the line, we can find a line tangent to the polygon and the tangent point should be in the lower side of the polygon, which is composed by the edges from the point with minimum $x_{i}$ to the point with maximum $x_{i}$ in counter-clockwise order. Define $E$ as the number of vertices in the lower side of the polygon, then these vertices can be represented by a sequence $S_{V_{1}}, S_{V_{2}}, \cdots, S_{V_{E}}$ beginning from the point with minimum $x_{i}$.

To investigate the tangency of a line and a polygon, the slopes of the edges of the polygon are analyzed and compared with the slope of the line $y=A x+B$. Based on the 
KKT condition (10), if the power $P_{V_{j}}$ makes at least one of the peak power constraints tight, e.g., the $s$-th constraint, its corresponding multiplier $\beta_{s}$ can be set to any value to make the line tangent to the polygon. If all the peak power constraints are not tight, the multipliers $\beta$ must be set to 0 , indicating that $B=0$.

Based on the above analysis, there can be three cases of possible optimal solutions.

Case 1: Only one user transmits when the line $y=A x+B$ is tangent to the polygon at a vertex in the lower side of the polygon.

Case 2: Two or more users transmit simultaneously when the line $y=A x+B$ is tangent to the polygon at an edge in the lower side of the polygon.

Case 3: The line $y=A x+B$ with the intercept at the original point is tangent to the polygon.

By comparing the objective functions of all possible optimal solutions, we obtain the optimal solution. Fig. 1 illustrates the three cases of possible optimal solutions, respectively.

In Case 1 , only one user transmits, so his transmit power $P_{i}$ should be set to the maximum value inside its feasible region.

$$
P_{V_{i}}=\max \left\{\frac{d_{1}}{b_{V_{i} 1}}, \frac{d_{2}}{b_{V_{i} 2}}, \cdots, \frac{d_{K}}{b_{V_{i} K}}\right\} .
$$

Since $A$ is dependent on $\mathbf{P}$ and only user $V_{i}$ transmits in that case, the slope of line $y=A x+B$ at $x_{V_{i}}$ can be denoted as $A\left(P_{V_{i}}\right)$. If $A\left(P_{V_{i}}\right)$ is between the slopes of line $S_{V_{i-1}} S_{V_{i}}$ and line $S_{V_{i}} S_{V_{i+1}}$,

$$
\frac{y_{V_{i}}-y_{V_{i-1}}}{x_{V_{i}}-x_{V_{i-1}}}<A\left(P_{V_{i}}\right)<\frac{y_{V_{i+1}}-y_{V_{i}}}{x_{V_{i+1}}-x_{V_{i}}} .
$$

The line is tangent to the polygon at $S_{V_{i}}$ and all the other points $S_{i} \in \mathcal{S}$ are above the line. Therefore, it is a local optimal solution that the user corresponding to $S_{V_{i}}$ transmits with the maximal power $P_{i}^{*}$.

In Case 2, multiple users transmit simultaneously when the line is tangent to the polygon and goes through all the corresponding points. As the polygon is external to all the points $S_{i} \in \mathcal{S}$, it is a convex polygon. In that case, the points corresponding to the transmitting users are neighbors at the edges of the polygon. If more than two users transmit simultaneously, these points must lie on a line. If multiple users transmit simultaneously, all the vertices of the feasible region are possible optimal solutions.

In Case 3, the intercept of the line $y=A x+B$ is the original point $O$, which is a fixed point. From the geometrical view in Fig. 1, at most two tangent lines of the polygon go through a fixed point. Therefore, at most two possible local optimal solutions in that case. Since $\beta=0$, it is not necessary to make some of the peak power constraints tight. The problem is degenerated to a simple one-dimensional optimum search problem in which the independent variable is the power of the user corresponding to the tangent points.

For the general power-control problem at each snapshot, it is assumed that the objective function $U(\mathbf{P})$ is an increasing function of $P_{i}$, so possible optimal solutions are achieved only when one or more constraints are tight. Case 3 can be considered as a subcase of Case 1 and Case 2, depending on how many points the line $y=A x+B$ goes through.

The geometry-based power-control optimization algorithm can be described as follows.

Geometry-based Power Control Algorithm:

Step 1: Check if the optimization problem can be modeled as a regular geometrical model based on the conditions in Theorem 3.

Step 2: Decompose $A x_{i}$ by using Strategy 1 and normalize $B$ by using Strategy 2 if necessary for constructing the regular geometrical model.

Step 3: For given $\lambda$, calculate the objective functions of possible optimal solutions in the three cases and compare them to obtain the optimal solution.

Step 4: Calculate the gap between the allocated power and average power constraints. If the current gap is smaller than the given threshold, stop the algorithm. Otherwise, adjust $\lambda$ using the steepest descent method, and repeat Step 3.

\section{B. Complexity Analysis}

Because there are both average and peak constraints in the optimization problem, two kinds of Lagrangian multipliers are needed to transform this problem to another optimization problem without any constraint. With the above optimization scheme utilizing the geometrical properties, the power vector $\mathbf{P}$ and the Lagrangian multipliers $\beta$ can be optimized in a deterministic manner for given $\lambda$ in the subproblem for each snapshot. The deterministic method lowers the computational complexity significantly compared to the classic numerical search method (e.g., the steepest descent method) for determining the Lagrangian multipliers. In addition, utilizing the properties of a geometrical model, the computational complexity of the power-control optimization problem can be reduced further; next, we will detail the complexity analysis.

Lemma 5: If $x$ users transmit simultaneously, at most $\frac{2(K+x) !}{x !(K+1) !}$ possible optimal solutions need to be considered for optimality.

Proof: The possible optimal solution appears at the vertices of the $x$-dimensional polytope feasible region only when $x$ users transmit simultaneously. The maximum number of vertices of the polytope is provided in [17].

According to the result in [14], at most two users transmit simultaneously for optimality with probability 1 if the coefficients in the general power-control optimization problem are random continuous variables, which is common in practical wireless networks. Here, we consider the complexity when at most two users transmit simultaneously, as stated in the following theorem.

Theorem 4: If at most two users transmit simultaneously, the computational complexity of the proposed geometry-based power-control algorithm is $O\left(N(K+N)^{2}\right)$.

Proof: The possible optimal solutions are at the vertices of the feasible region. Thus, we should first obtain the vertices 
of the feasible region, and then calculate the utility functions of the vertices.

For the calculation of vertices, there are $K$ peak constraints and $P_{i} \geq 0, \forall i \in \mathcal{N}$, so the total number of peak constraints should be considered as $K+N$ here. If only one user transmits as in Case 1, at most $N$ possible optimal solutions need to be considered. If two users transmit simultaneously as in Case 2, the $N$-dimensional polytope feasible region degenerates to the two-dimensional polygon region for each pair of users. Considering these two constraints can obtain a possible vertex of the feasible region, so there are $C_{K+N}^{2}=(K+N)(K+N-1)$ pairs of constraints for two simultaneously transmitting users. Since the points corresponding to the two simultaneously transmitting users are neighbors at the edges of the polygon, at most $N-1$ cases are considered. The computational complexity of Case 3 can be ignored because only 2 possible optimal solutions exist, even if this case is not included in Case 1 and Case 2. The complexity for calculation of vertices is $O\left(N(K+N)^{2}\right)$.

For the calculation of utility functions, there are at most $(N-1)(K+2)$ possible optimal solutions when $x=2$ according to Lemma 5. The complexity is $O(N K)$.

Combining the complexities of both parts, the total worstcase computational complexity is $O\left(N(K+N)^{2}\right)$.

Remark 3: Without considering the properties of a geometrical model, the conventional algorithm finds the vertices of the $N$-dimensional feasible region. For the calculation of vertices, there are $C_{K+N}^{N}$ combinations with the complexity $O\left(2^{K+N}\right)$. Solving $N$-dimensional linear equations incurs $O\left(N^{3}\right)$ computational cost by using Gaussian Elimination for each combination [18]. For the calculation of utility functions, according to Lemma 5, there are $\frac{2(K+N) !}{N !(K+1) !}$ vertices with complexity $O\left(2^{K+N}\right)$. When only two users transmit simultaneously, the complexity of calculation of utility functions reduces to $O(N K)$. Combining both parts, the total complexity of the conventional algorithm is $O\left(N^{3} 2^{K+N}\right)$.

Fig. 2 compares the complexity of the proposed algorithm and that of the conventional algorithm using the number of operations during the calculation of both vertices and utility functions. The complexity of utility calculation is dependent on the utility function. It is assumed to be $N$ operations in the numerical analysis for simplicity. From the results, the computational complexity of the proposed geometry-based algorithm is much lower than the conventional algorithm, and increases slowly with the increase of the number of users $N$ and the number of peak constraints $K$. One of the advantages of the proposed algorithm is degenerating the $N$-dimensional polytope feasible region to multiple two-dimensional polygon regions, and thus reduce the computational complexity. It cannot reduce the complexity when $N=2$, so an exception exists.

According to the characteristics of the geometrical model, the global optimal solution can be obtained directly for the concave utility functions. The existence and uniqueness of the optimal solution are obtained as stated in the following theorem.

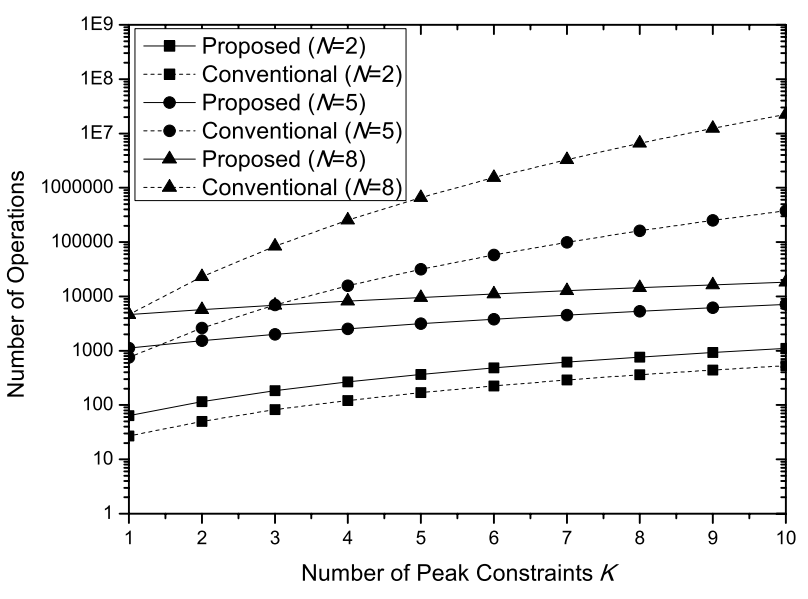

Fig. 2. Complexity comparison

Theorem 5: A unique optimal solution exists if $A$ is decreasing monotonically in $x$.

Proof: The slopes of the edges in the counter-clockwise order increase from $-90^{\circ}$ to $90^{\circ}$ monotonically at the lower side of the polygon. Let $L$ be a piece-wise curve composed with the slopes of the edges at the lower side of the polygon and $M$ be a folding line composed by the slopes of line $y=$ $A x+B$. If $A$ is decreasing monotonically in $x$, there exists a unique intersection between $M$ and $L$ exists, so does the unique solution.

Remark 4: In this case, the optimal transmitting users can be determined directly by comparing $L$ and $M$ at the points $S_{V_{i}}, \forall i \in\{1,2, \cdots, E\}$, which results in low complexity.

\section{Discussion on IrRegular Geometrical Models}

There are two possible reasons to make the geometrical model irregular. First, $x_{i}$ changes with different P's. Second, $B$ is not unique for different $i$ 's. For the latter case, an alternative way is to set $B$ to 0 and place its expression in $y_{i}$ as

$$
y_{i}=\sum_{j} \lambda_{j} a_{i j}+\sum_{k} \beta_{k} b_{i k} .
$$

This way, the non-unique $B$ can be transformed to $y_{i}$ which varies with the independent variable $\beta$. Therefore, compared to the regular geometrical model, the main challenge associated with the irregular geometrical model is that the position of $S_{i} \in \mathcal{S}$ varies with different $\mathbf{P}$ 's. In such a case, when the independent variable $\mathbf{P}$ is adjusted, the positions of both the line $y=A x+B$ and the points $S_{i} \in \mathcal{S}$ change, making the irregular geometrical model difficult to find the optimal solution and have the same-order computational complexity as the conventional methods.

Here, we define a condition for the subset of irregular geometrical models which can adopt the proposed geometrybased power-control scheme.

Definition 2 (Monotonic Distance Condition): The distance between the line $y=A x+B$ and the point $S_{i}$ increases with the increase of $P_{i}$. 


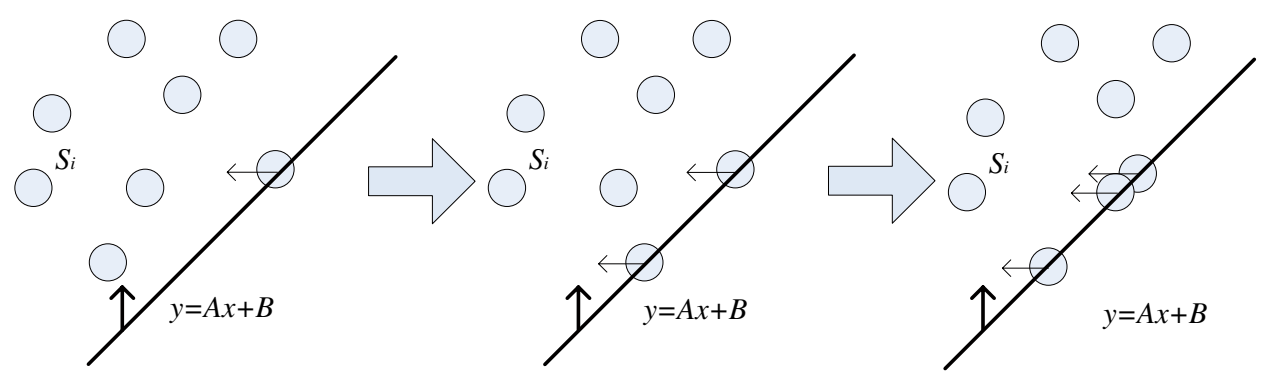

Fig. 3. An example irregular geometrical model

For the cases in which the monotonic distance condition is satisfied, the point $S_{i}$ tries to move far away from the line $y=$ $A x+B$ when the power $P_{i}$ increases. According to Theorem 2 , the points corresponding to transmitting users should just be on the line $y=A x+B$. To keep the line goes through the point $S_{i}$, we continue to move the line for tracking the point by adjusting $\beta$. The proposed geometry-based power-control scheme can be deployed with some modifications in Step 3.

Modification for Irregular Geometrical Model:
1. The positions of the points in $\mathcal{S}$ are initially set to
the case of $P_{i}=0, \forall i \in \mathcal{N}$. Find the tangent point
of the line and the external polygon composed by all
the points in $\mathcal{S}$. Add the user corresponding to the
tangent point into $\mathcal{N}_{T}$.
2. Increase the power of user $i, i \in \mathcal{N}_{T}$, whose
corresponding point is on the line. Adjust $\mathbf{P}$ and $\beta$
for to make the line always goes through all these
points $S_{i}, \forall i \in \mathcal{N}_{T}$.
3. If the line goes through another point, add the
corresponding user into $\mathcal{N}_{T}$ and increase its power
along with other users with non-zero power.
4. If all the users on the line cannot increase power
any more without violating the constraints, stop the
algorithm. The users corresponding to the points on
the line should transmit simultaneously.

Fig. 3 provides an example procedure of geometry-based optimal power-control for the irregular geometrical model.

The utility function $U(\mathbf{P})$ of the general power-control optimization problem is an increasing concave function of $\mathbf{P}$, so $\partial U(\mathbf{P}) / \partial P_{i}$ is decreasing with $P_{i}$. Obviously, with an increase of $P_{i}$, the distance between the line $y=A x+B$ and the point $S_{i}$ increases. Therefore, the geometry-based power-control scheme is always suitable for the general powercontrol optimization because the monotonic distance condition is satisfied.

\section{EXAMPLES}

In this section, we discuss two power-control problems with known optimal solutions as examples, providing an insight into the deployment of the proposed geometry-based scheme.

Example 1 (Multiple access channels with average and peak power constraints [14]):

$$
\begin{aligned}
\max & U(\mathbf{P})=\mathbb{E}\left[\frac{1}{2} \log \left(1+\frac{\sum_{i=1}^{N} P_{i} g_{i}}{\sigma^{2}}\right)\right] \\
\text { s.t. } & P_{i} \geq 0, \forall i \in \mathcal{N} \\
& \mathbb{E}\left[P_{i}\right] \leq P_{i}^{\max }, \forall i \in \mathcal{N} \\
& \sum_{i \in \mathcal{N}} P_{i} h_{i} \leq Q
\end{aligned}
$$

where $U(\mathbf{P})$ is represented by the sum-rate of multiple access channels, $Q$ is the interference limit at the measuring point, $\sigma^{2}$ is the power of thermal noise, $g_{i}$ and $h_{i}$ are the path gains from user $i$ to the receiver and the measurement point, respectively.

By checking the two conditions of Theorem 3, this problem is found to be solvable by using a regular geometrical model. To normalize $B$ under Strategy 2 for constructing the regular geometrical model, we define $\omega_{j}=P_{j} h_{j} / Q$, which is considered as a new independent variable, instead of $P_{j}$. The partial derivative of the Lagrangian function with respect to $\omega_{j}$ is

$$
\frac{\partial L}{\partial \omega_{j}}=\frac{Q g_{j} / h_{j}}{1+\sum_{i \in \mathcal{N}} \omega_{i} Q g_{i} / h_{i}}-\lambda_{j} Q / h_{j}-\beta .
$$

By using Strategy 1, we found the denominator part of $\partial U / \partial \omega_{j}$ is symmetric for all $\omega_{j}$. Accordingly, $A$ and $x_{i}$ is decomposed as the approach in Remark 2. The coefficients of the regular geometrical model are listed as:

$$
\begin{gathered}
x_{j}=Q g_{j} / h_{j} \\
y_{j}=\lambda_{j} Q / h_{j} \\
A=\frac{1}{1+\sum_{i \in \mathcal{N}} \omega_{i} Q g_{i} / h_{i}} . \\
B=-\beta .
\end{gathered}
$$

To find the optimal solution, the power $\mathbf{P}$ is adjusted to make the line tangent to the polygon. One or two users can transmit simultaneously. This regular geometric model is found to satisfy the condition in Theorem 5, so the optimal solution can be obtained directly with a low computational complexity.

Remark 5: Due to the duality of multiple access channels and broadcast channels [19], the proposed geometry-based power-control scheme can also be adopted for broadcast channels. 
Example 2 (Multiple parallel channels with peak total and and individual power constraints [20], [21]):

$$
\begin{array}{ll}
\max & U(\mathbf{P})=\sum_{i \in \mathcal{N}} \log \left(1+\frac{P_{i} g_{i}}{\sigma^{2}}\right) \\
\text { s.t. } & P_{i} \geq 0, \forall i \in \mathcal{N} \\
& P_{i} \leq P_{i}^{\max }, \forall i \in \mathcal{N} \\
& \sum_{i \in \mathcal{N}} P_{i} \leq \bar{P}
\end{array}
$$

where $U(\mathbf{P})$ is represented by the total throughput of $N$ parallel channels.

The partial derivative of Lagrangian function with respect to $P_{i}$ is

$$
\frac{\partial L}{\partial P_{i}}=\frac{g_{i}}{N_{0}+P_{i} g_{i}}-\beta-\sum_{k \in \mathcal{N}} \beta_{k}
$$

From the expression of the derivative, it is an irregular geometrical model by checking the two conditions in Theorem 3 . As $\partial U(\mathbf{P}) / \partial P_{i}$ does not have any symmetric part for all $P_{i}$, we set $A=1$ and $x_{i}=\partial U(\mathbf{P}) / \partial P_{i}$. Since there exist only peak constraints, $y_{i}=0, \forall i$, indicating that all the points lay on a horizonal line. The geometrical power-control model can be written as

$$
\begin{gathered}
x_{i}=\frac{g_{i}}{N_{0}+P_{i} g_{i}} \\
y_{i}=0 \\
A=1 \\
B=-\beta-\sum_{k \in \mathcal{N}} \beta_{k} .
\end{gathered}
$$

From the geometrical model, one can observe that the slope of the line $y=A x+B$ is 1 and $y_{i}$ for all $i \in \mathcal{N}$ is 0 . By the proposed geometry-based optimal power-control algorithm for the irregular geometrical model, the users' power values increase with the line $y=A x+B$ going through the points which correspond to the transmitting users. In such a case, the partial derivatives $\partial U(\mathbf{P}) / \partial P_{i}$ are equal to 0 for the transmitting users. The algorithm stops when all the users cannot increase their power. The result is consistent with the water-filling power allocation with the limits for individual users in [20], [21].

\section{CONCLUSIONS}

In this paper, we have generalized the geometry-based optimal power-control scheme to a larger class of powercontrol optimization problems in wireless networks, including multiple access channels, broadcast channels, and multiple parallel channels. Computational complexity is reduced significantly by exploiting the geometrical properties on the position relationship between the line $y=A x+B$ and the points $S_{i} \in \mathcal{S}$.

For construction of a regular geometrical model, we have proposed strategies for the decomposition of $A$ and $x_{i}$ and the normalization of $B$. If the two conditions are satisfied, the power-control optimization problem can be modeled as a regular geometrical model using the two construction strategies. For the optimization of regular geometrical model, the line is adjusted to be tangent to the polygon composed by the points $S_{i} \in \mathcal{S}$. The computational complexity is $O\left(N(K+N)^{2}\right)$, which is much lower than that of the conventional algorithm. In addition, some modifications are made to the proposed geometry-based algorithm for irregular geometrical models. Finally, two examples are provided for deployment of the proposed scheme.

\section{REFERENCES}

[1] J. Zander, "Performance of optimum transmitter power control in cellular radio systems", IEEE Trans. Veh. Technol., vol. 41, no. 1, pp. 57-62, Feb. 1992

[2] R. D. Yates, "A framework for uplink power control in cellular radio systems", IEEE J. Sel. Areas Commun., vol. 13, no. 7, pp. 1341-1347, Sep. 1995

[3] D. Goodman, N. Mandayam, "Power Control for Wireless Data", IEEE Personal Commun., vol. 7, no. 2, pp. 48-54, Apr. 2000

[4] C.W. Sung, K.K. Leung, "A generalized framework for distributed power control in wireless networks", IEEE Trans. Info. Theory, vol. 51, no. 7 , pp. 2625-2635, Jul. 2005

[5] P. Patel, J. Holtzman, "Analysis of a simple successive interference cancellation scheme in a DS/CDMA system", IEEE J. Sel. Areas Commun., vol. 12, no. 5, pp. 796-807, Jun. 1994

[6] C.Y. Wong, R.S. Cheng, K.B. Letaief, R.D. Murch, "Multiuser OFDM with adaptive subcarrier, bit, and power allocation", IEEE J. Sel. Areas Commun., vol. 17, no. 10, pp. 1747-1758, Oct. 1999

[7] G.J. Goschini, "Layered space-time architecture for wireless communication in a fading environment when using multi-element antennas", Bell Labs Technical Journal, vol. 1, no. 2, pp. 41-59, 1996

[8] M. Chiang, P. Hande, T. Lan, C.W. Tee, "Power Control in Wireless Cellular Networks", Foundations and Trends in Networking, vol. 2, no. 4, pp. 381-533, Apr. 2008

[9] M. Schubert, H. Boche, "Solution of the multiuser downlink beamforming problem with individual SINR constraints", IEEE Trans. Veh. Technol., vol. 53, no. 1, pp. 18-28, Jan. 2004.

[10] W. Yu, T. Lan, "Transmitter optimization for the multi-antenna downlink with per-antenna power constraints", IEEE Trans. Signal Process., vol. 55, no. 6, pp. 2646-2660, Jun. 2007

[11] S. Boyd, L. Vandenberghe, Convex Optimization, Cambridge Univ. Press, 2004

[12] C. Zeng, L. M. C. Hoo, J.M. Cioffi, "Efficient water-filling algorithms for a Gaussian multiaccess channel with ISI", Proc. of IEEE VTC 2000, pp. 1072-1077, 2000

[13] W. Wang, T. Peng, W. Wang, "Optimal power control under interference temperature constraints in cognitive radio network", Proc. of IEEE WCNC 2007, Mar. 2007

[14] W. Wang, W. Wang, Q. Lu, K.G. Shin, T. Peng, "Geometry-based Optimal Power Control of Fading Multiple Access Channels for Maximum Sum-rate in Cognitive Radio Networks", IEEE Trans. Wireless Commun., vol. 9, no. 6, pp. 1843-1848, Jun. 2010

[15] D. Tse, S. Hanly, "Multiaccess fading channels-Part I: Polymatroid structure, optimal resource allocation and throughput capacities", IEEE Trans. Info. Theory, vol. 44, no. 7, pp. 2796-2815, Nov. 1998

[16] K. Kar, S. Sarkar, L. Tassiulas, "A simple rate control algorithm for maximizing total user utility", Proc. of IEEE INFOCOM 2001, Apr. 2001

[17] T.L. Saaty, "The number of vertices of a polyhedron", The American Mathmatical Monthly, vol. 62, no. 5, pp. 326-331, May 1955

[18] T.H. Cormen, C.E. Leiserson, R.L. Rivest, C. Stein, Introduction to Algorithms (Third Edition). MIT Press. 2001

[19] S. Vishwanath, N. Jindal, A. Goldsmith, "On the duality of Gaussian multiple-access and broadcast channels", IEEE Trans. Inform. Theory, vol. 50, no. 5, pp. 768-783, May 2004.

[20] W. Wang, W. Wang, Q. Lu, T. Peng, "An Uplink Resource Allocation Scheme for OFDMA-Based Cognitive Radio Networks", Wiley Int. J. of Commun. Sys., vol.22, no. 5, pp. 603-623, May 2009

[21] W. Wang, K.G. Shin, W. Wang, "Distributed resource allocation based on queue-balancing in multi-hop cognitive radio networks", IEEE/ACM Trans. Networking, to appear 